\title{
Ein neuer Papyrus aus dem Dossier des comes Johannes
}

\section{(P.Lips. inv. 1354)}

\author{
Tafel VI
}

Bärbel Kramer (Trier) und Dieter Hagedorn (Köln)

\begin{abstract}
Edition of a new papyrus from the dossier of the comes Johannes, containing the order of the comes to Philoxenos, manager of a hamlet or estate called Peribleptou, to supply 12 nomismatia for the purchase of reed for the vineyard and 4 nomismatia for bulls.
\end{abstract}

Keywords: comes Johannes, Philoxenos, Peribleptou, order to pay

Im Jahre 2004 haben wir in dieser Zeitschrift die Erstedition einer kleinen, aus fünf Texten bestehenden Gruppe von Papyri vorgelegt, vermehrt um den Wiederabdruck eines verwandten, aber schon länger bekannten Texts, ${ }^{1}$ deren Gemeinsamkeit es ist, daß es sich bei ihnen um Auszahlungsanweisungen handelt, die ein comes namens Johannes in den Jahren zwischen 472 und 481/2 n.Chr. an verschiedene Verwalter seiner Besitzungen im Oxyrhynchites gerichtet hat. Die Originale der uns damals bekannten Stücke gehören zu den Beständen der Sammlungen in Hamburg, Heidelberg und Birmingham (P.Harris). Nadine Quenouille ist nun im Zuge ihrer Mitarbeit an dem „Papyrus-Projekt Halle-Jena-Leipzig“ auf eine weitere solche Auszahlungsanweisung aufmerksam geworden, die demselben Kleinarchiv zuzurechnen ist. Wir danken Frau Quenouille sehr herzlich dafür, daß sie uns die Bearbeitung des neuen Texts angeboten hat, sowie Reinhold Scholl für die Publikationserlaubnis.

P.Lips. inv. 1354 weist exakt dieselben formalen Eigentümlichkeiten auf, die wir seinerzeit in der Einleitung unseres Aufsatzes beschrieben haben. Der Haupttext ist auf dem Rekto des schmalen Papyrusblattes transversa charta, also im Querformat und gegen den Faserverlauf, geschrieben worden, und die Verteilung der Textelemente (Absender, Adressat, Auszahlungsanweisung, Gegenzeichnung, Datierung) entspricht vollkommen der Skizze, die wir dort auf S. 160 abgedruckt haben. Auf dem Verso steht parallel zum Faserverlauf eine einzige Zeile mit einer Inhaltsangabe.

${ }^{1}$ D. Hagedorn, B. Kramer, Fünf neue Papyri des comes Johannes (P.Hamb. Inv. 532, 533, 538, 547 und P.Heid. inv. $1800+1843$ ) und Neuabdruck von P.Harris I 91, APF 50/2 (2004) 158-171 mit Taf. X-XIV. 
Der Empfänger der neuen Anweisung, Philoxenos, der Verwalter eines Weilers oder Gutes mit der Bezeichnung „Peribleptu“, ist uns schon aus P.Hamb. inv. 533 bekannt, einer Anweisung vom 8. Sept. 473 n.Chr., die wir in unserem früheren Aufsatz unter der Nummer 3 veröffentlicht haben. Der neue Text ist nur knapp fünf Monate später entstanden, nämlich am 31. Januar 474. Der Schreiber, der den Haupttext geschrieben hat (d.h. die 1. Hand), ist ganz offenkundig in beiden Anweisungen derselbe, ebenso der Schreiber der Gegenzeichnung (2. Hand), von dem im übrigen wohl auch die Gegenzeichnungen in den Nummern 1, 2 und in P.Harris I 91 stammen und in dem wir möglicherweise den comes Johannes selbst sehen dürfen (vgl. APF 50 [2004] S. 160).

Johannes erteilt in P.Lips. inv. 1354 seinem Verwalter Philoxenos den Auftrag, für den Ankauf von Rohr, welches der Anfertigung von Stützen für die Reben des Weingutes dienen soll, zwölf Goldsolidi bereitzustellen. Die Anweisung datiert in die 12. Indiktion, aber die Arbeiten selbst sollen erst in der bevorstehenden 13. Indiktion durchgeführt werden. Die Menge des zu beschaffenden Rohres wird nicht beziffert, doch verdeutlicht die nicht unbeträchtliche Summe von zwölf Solidi, daß das Weingut einen erheblichen Bedarf an Rohr hatte und entsprechend ausgedehnt gewesen sein muß.2 Seltsam erscheint, daß in der Inhaltsangabe auf der Rückseite außer von den zwölf Solidi für den Preis des Rohrs auch noch von vier Solidi $\dot{v} \pi(\grave{\varepsilon} \rho) \tau \mu(\hat{\eta} \varsigma) \tau \alpha v ́ \rho(\omega v)$ die Rede ist. Darüber, wie dies zu verstehen ist, läßt sich nur spekulieren. Die Angabe könnte ganz einfach versehentlich auf der falschen Auszahlungsanweisung angebracht worden sein. Sollte sie aus einem uns nicht nachvollziehbaren Grund ihre Berechtigung gehabt haben, wäre zu fragen, ob die Rinder möglicherweise als Arbeitstiere im Zusammenhang mit der Anlage der Spaliere im Weinberg zum Einsatz kommen sollten oder ob keinerlei sachliche Verbindung bestand.

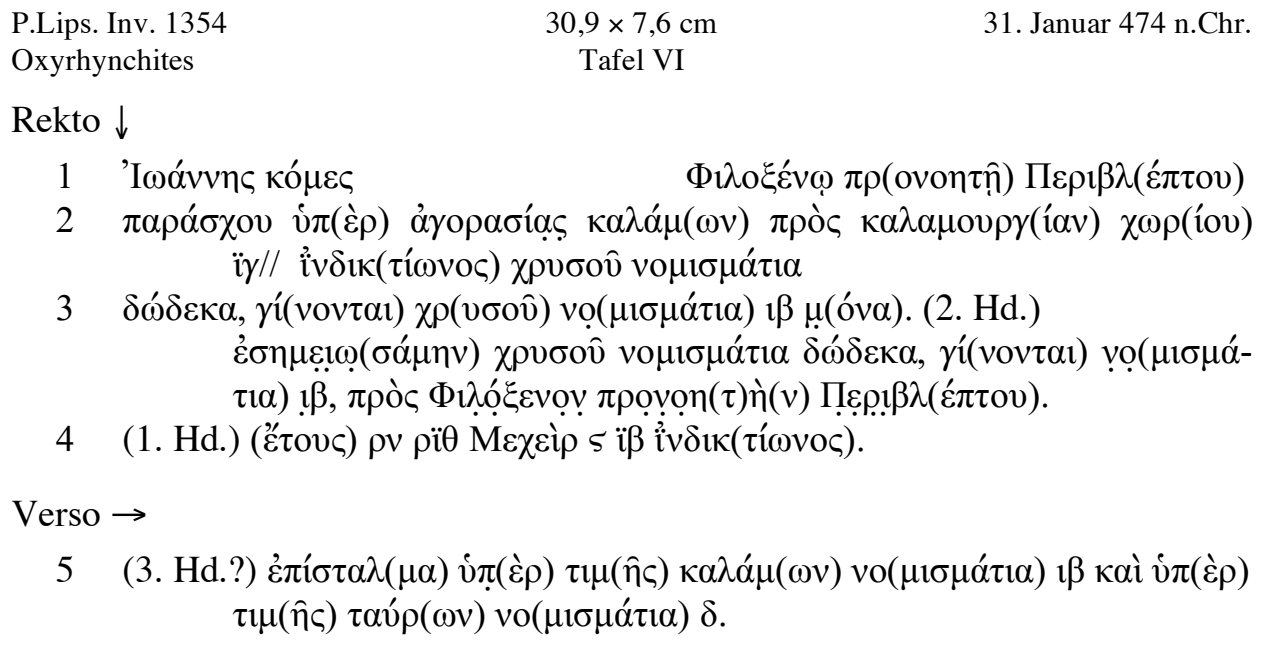

${ }^{2}$ Vgl. unten den Zeilenkommentar zu Z. 2. 


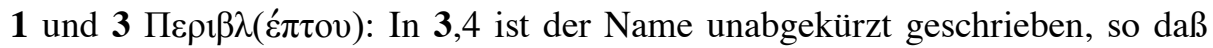
die Auflösung sicher ist. Wir kennen das Anwesen bislang nur aus diesen beiden Texten ( $\mathbf{3}$ und dem vorliegenden Papyrus).

2 vं $\pi(\varepsilon \rho)$ : Der )-förmige Haken, der bekanntlich eine sehr übliche Abkürzung für $\pi\left(\right.$ ) ist, steht hier nicht hinter, sondern über demYpsilon, also $\vartheta^{2}$. Ebenso hat in 3 derselbe Schreiber das Wort $\dot{v} \pi(\dot{\varepsilon} \rho)$ in Z. 3 und (besonders klar und deutlich) in Z. 6 (Verso) geschrieben; vgl. die Abbildungen in APF 50 (2004) Tafel XIII.

Zur Bedeutung des Wortes $\chi \omega$ píov s. R.S. Bagnall, The Date of P.Kell. I G. 62 and the Meaning of $\chi \omega$ píov, CE 74 (1999) 329-333. In Anbetracht des Zusam-

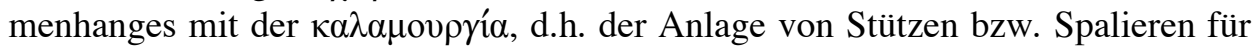
Weinstöcke, wird deutlich, daß hier die Bedeutung „Weingut, Weinpflanzung“ vorliegt.

$\mathrm{Da}$ im holzarmen Ägypten zur Stützung der Reben in erster Linie Rohr verwendet wurde, machen unsere Quellen allenthalben deutlich und ist in der einschlägigen Literatur schon vielfach dargestellt worden. ${ }^{3}$ In welcher Weise man das Rohr dabei eingesetzt hat, beschreiben manche Pachtverträge über Weingärten detailliert, so etwa P.Soterichos 1 und 2 (Theadelphia; 69 bzw. 71 n.Chr.) und P.Flor. III 369 (Hermupolis; 139 oder 149 n.Chr.); man vergleiche S. Omars Kommentar zu P.Soterichos 2,22-23. In der Regel legten die Winzer eigene Rohrpflanzungen in unmittelbarer Nähe der Weingärten an, um sich so selbst mit dem benötigten Material versorgen zu können. ${ }^{4}$ Sofern dies unterblieb oder die eigene Produktion nicht ausreichte, war Ankauf oder Zukauf von Rohr erforderlich. ${ }^{5}$

Leider erfahren wir in der vorliegenden Anweisung weder, wieviel Stück Rohr für die 12 bereitgestellten Solidi erwartet wurden, noch für welche Fläche der Weinkulturen der Betrag ausreichen sollte. Andere spätantike Texte liefern jedoch Vergleichszahlen: Laut P.Oxy. XVI 1911,175-177 (Oxy.; 557 n.Chr.) wurden im

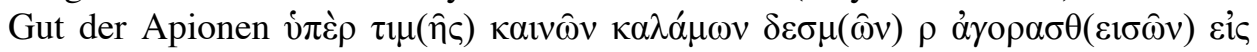

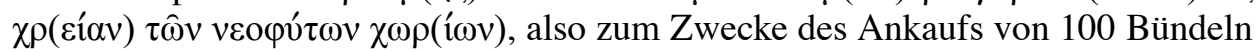

\footnotetext{
${ }^{3}$ Wir verweisen nur auf M. Schnebel, Die Landwirtschaft im hellenistischen Ägypten (Münchener Beiträge zur Papyrusforschung und antiken Rechtsgeschichte 7), München 1925, S. 254 262; C. Ricci, La coltura della vite e la fabbricazione del vino nell'Egitto greco-romano (Studi della Scuola Papirologica IV 1), Milano 1924, S. 26f. und 34-39; K. Ruffing, Weinbau im römischen Ägypten (Pharos. Studien zur griechisch-römischen Geschichte 12), St. Katharinen 1999, S. 54-70 (Rohranbau) und 140-145 (Anlage und Pflege der Rebunterstützungen).

${ }^{4}$ Belege bei Ruffing (wie Anm. 3) in den Listen auf S. 54-57.

5 Dies veranschaulicht schön SB XXIV 16323, ein Brief aus dem Heroninos-Archiv, in dem sich auch die größte in den Papyri erwähnte Menge von Rohr findet, nämlich 90.000 Stück, die bereits herbeigeschafft waren; da aber in den Weingärten $(\kappa \tau \eta \dot{\mu} \mu \alpha \tau \alpha)$ selbst kein Rohr vorhanden war, verlangt Heroninos weitere 40.000 Stück oder noch mehr, die angekauft werden mußten

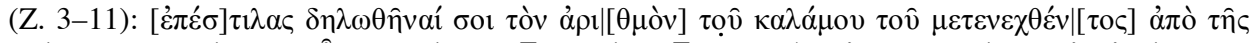

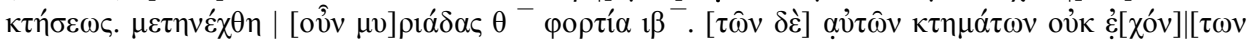

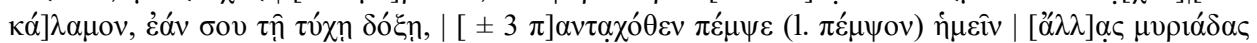

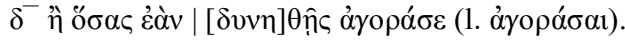


Rohr (vielleicht $=5.000$ Stück $^{6}$ ), die bei der Neuanlage von Weinpflanzungen im Umfang von 1 1 $\frac{1}{2}$ Aure Verwendung finden sollten, 2 Solidi minus 9 Keratia verausgabt. Zu vergleichen ist damit auch P.Oxy. XVI 1912,152 (Oxy.; vor 566

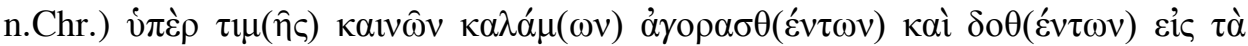

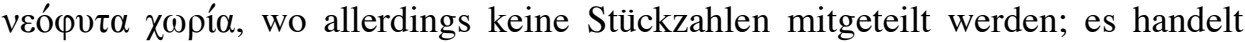
sich jedoch um $5 \frac{1}{2}$ zu bearbeitende Aruren, und pro Arure werden 2 Nomismata berechnet, insgesamt 11 Nomismata minus 391/2 Keratia. In BGU XII 2208 (Hermupolis; 614 n.Chr.), einem Lieferungskauf von 134 Bündeln Rohr, wird vereinbart, daß der Lieferant, der den (nicht bezifferten) Kaufpreis schon erhalten hat, 4 Solidi zu zahlen habe, falls er der Lieferungsverpflichtung nicht nachkomme; in dem Betrag dürfte also ein Strafzuschlag enthalten sein. ${ }^{7}$ Natürlich schwankten die Preise, und zwar nicht unbeträchtlich. So wird in VBP IV 95 (Hermopolites; Anfang 6. Jh., vgl. BL IX 13) in Z. 233 und 234 für 8.000 Stück Rohr ein Preis von 1 Nomisma minus 6 Keratia angesetzt, in Z. 114 dagegen gilt derselbe Preis für nur 7.000 Stück, aber in Z. 113 für 9.500 Stück; vgl. die Korrekturen von F. Morelli in ZPE 112 (1998) 141. Immerhin dürfen wir folgern, daß die 12 Solidi, welche der comes Johannes hier bereitstellt, für einen Weinberg von ungefähr 6 Aruren ausgereicht haben könnten.

Eine direkte Parallele zu dem hier zugrunde liegenden Vorgang (Bereitstellung von Geld zum Zwecke des Ankaufs von Rohr für Arbeiten im Weinberg) beschreibt die Quittung CPR XIX 32 (erweiterte Neuedition von CPP XX 240; Arsi-

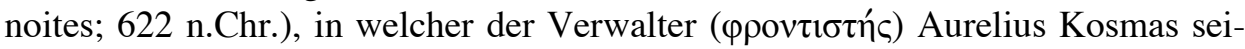
nem Herrn, dem Stratelates und Pagarchen Flavius Menas, bestätigt (Z. 9-18):

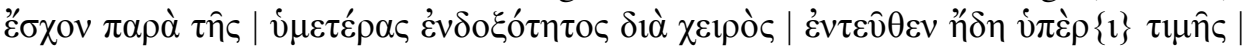

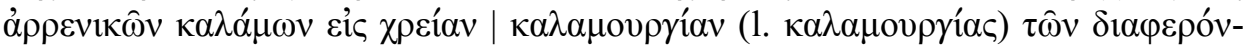

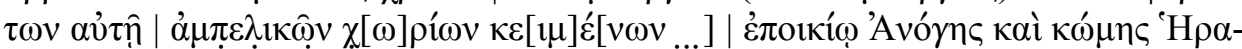

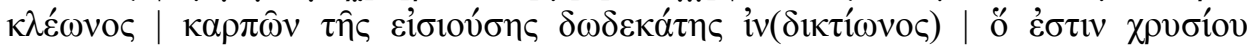

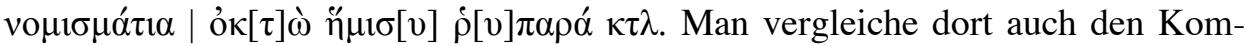
mentar.

3 Das Ende der Gegenzeichnung ist mit extremen Verschleifungen geschrieben und hat sich nur dank der Parallelen, zumal der von 3,4, entziffern lassen.

\footnotetext{
${ }^{6}$ Ein Bündel Rohr setzte sich - zumindest in römischer Zeit - anscheinend standardmäßig aus 50 Stück zusammen; dies ergibt sich aus P.Mil. Vogl. II 69 B 52-53 = P.Mil. Vogl. VII 308,51-52 (beide Tebtynis; 2. Jh. n.Chr.), wo man den Kommentar vergleiche.

${ }^{7}$ In anderen Lieferungskäufen von Rohr finden sich überhaupt keine Preisangaben; s. BGU III 837 (Arsinoiton Polis; 609 n.Chr.), BGU XII 2210 (Hermupolis; 617 n.Chr.) und P.Rain. Unterricht 112 (griechisch-koptischer Mustervertrag; Hermupolis; 8. Jh.). In der „Quittung im Zusammenhang mit einer Lieferung von Schilfrohr" SPP III 67 (Herakleopolites; 7. Jh.) ist der Preis für

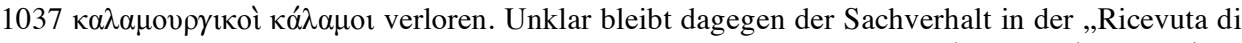


(Z. 3) die Rede, die auf einen (schon gezahlten?) Solidus irgendwie angerechnet werden sollen




5 Auch P.Harr. II 232 (Oxyrhynchites, 304 n.Chr.), eine Anweisung an einen

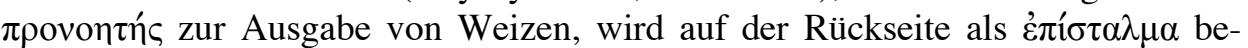
zeichnet. (Vgl. auch P.Grenf. I 63.)

\section{Übersetzung}

(1. Hd.) „Comes Johannes an Philoxenos, den Verwalter von Peribleptu. Zahle aus für den Kauf von Rohr zum Zwecke der Anbringung von Weinspalieren im (Wein)gut für die 13. Indiktion zwölf Goldnomismatia, macht 12 Goldnomismatia, sonst nichts.“ (2. Hd.) „Ich habe unterzeichnet zwölf Goldnomismatia, macht 12 Nomismatia, für Philoxenos, den Verwalter von Peribleptu.“ (1. Hd.) „Im Jahr 150/119, 6. Mecheir, 12. Indiktion.“

Verso: (3. Hd.?) ,Zahlungsanweisung für den Kaufpreis von Rohr 12 Nomismatia und für den Kaufpreis von Stieren 4 Nomismatia.“ 